Editor's Note: These short reviews of a recent paper in the Journal, written exclusively by graduate students or postdoctoral fellows, are intended to mimic the journal clubs that exist in your own departments or institutions. For more information on the format and purpose of the Journal Club, please see http://www.jneurosci.org/misc/ifa_features.shtml.

\title{
Endocannabinoid-Dependent Regulation of Feedforward Inhibition in Cerebellar Purkinje Cells
}

\author{
Cameron H. Good \\ Department of Health and Human Services, National Institutes of Health, National Institute on Drug Abuse Intramural Research Program, Cellular \\ Neurobiology Branch, Electrophysiology Research Unit, Baltimore, Maryland 21224 \\ Review of Beierlein and Regehr (http://www.jneurosci.org/cgi/content/full/26/39/9935)
}

The discovery of endogenous cannabinoids (eCBs) in the brain has opened up new avenues of research and provided us with novel mechanisms in which postsynaptic neurons can regulate their own synaptic inputs. Endocannabinoids are released from postsynaptic cells in a calcium-dependent manner after brief depolarizing input. Once released, eCBs inhibit neurotransmitter release via the retrograde activation of cannabinoid CB1 receptors (CB1Rs) located on axon terminals (Wilson and Nicoll, 2001).

In the cerebellum, the regulation of Purkinje cell (PC) activity is of interest, because their axons form the sole output of this structure and because of the role that these neurons play in the acquisition and refinement of motor skills. PCs receive excitatory input from climbing fibers originating in the inferior olive, and from granule cell parallel fibers (PFs). PCs receive inhibitory inputs from local interneurons such as basket (BCs) and stellate cells (SCs) (Fig. 1) (Eccles et al., 1967). Although it is well known that PCs and other principal neurons release eCBs, the

Received Nov. 6, 2006; revised Nov. 15, 2006; accepted Nov. 16, 2006.

This work was supported by the National Institutes of Health, Department of Health and Human Services, and the National Institute on Drug Abuse-Intramural Research Program.

Correspondence should be addressed to Cameron H. Good, Electrophysiology Research Unit, Cellular Neurobiology Branch, National Institute on Drug Abuse, Intramural Research Program, 5500 Nathan Shock Drive, Baltimore, MD 21224.E-mail: goodc@mail.nih.gov.

D01:10.1523/JNEUROSCI.4842-06.2007

Copyright $\odot$ 2007 Society for Neuroscience $\quad$ 0270-6474/07/270001-03\$15.00/0 role of GABAergic interneurons in retrograde eCB signaling is poorly understood. Beierlein and Regehr (2006) have made a significant contribution to the field by showing that BCs and SCs can release eCBs and thereby regulate their synaptic inputs.

Previously, eCB release from interneurons was examined in the hippocampus (Hoffman et al., 2003) and neocortex (Bacci et al., 2004) with mixed results. Whole-cell recordings from hippocampal stratum radiatum and stratum oriens interneurons revealed that synaptic GABAergic inputs were inhibited by the cannabinoid agonist $(R)-(+)-[2,3-$ dihydro-5-methyl-3-(4-morpholinylmethyl)pyrrolo[1,2,3-de]-1,4-benzoxazin-6-yl]-1-napthalenylmethanone (WIN55,212-2), whereas glutamatergic inputs were unaffected (Hoffman et al., 2003). This contrasted with CA1 pyramidal neurons in which both GABAergic and glutamatergic inputs were inhibited by WIN55,212-2. eCBs can be released from CA1 pyramidal neurons via somatic depolarization, where they can then retrogradely act to inhibit their own GABAergic inputs (Wilson and Nicoll, 2001). Although this depolarization-induced suppression of inhibition (DSI) was seen in pyramidal neurons, it was not observed in the interneurons in this study (Hoffman et al., 2003). This demonstrated that, whereas GABAergic inputs to hippocampal interneurons were inhibited by WIN55,212-2, these cells appeared unable to release eCBs (Hoffman et al., 2003). In contrast, a study in neocortical GABAergic interneurons found that lowthreshold-spiking cells released eCBs that inhibited these neurons by initiating a long-lasting hyperpolarization of the membrane potential via CB1Rs (Bacci et al., 2004). This form of eCB-dependent autoinhibition was unique, because previously these molecules were found only to act at presynaptic sites as retrograde messengers. Interestingly, the same protocol tested in fast-spiking interneurons revealed no change in membrane potential, further suggesting heterogeneity in the release of eCBs from distinct interneuron populations (Bacci et al., 2004). It is in this context that the recently published study by Beierlein and Regehr (2006) examined the mechanisms through which distinct neuronal populations in the cerebellumreleased eCBs.

Previous studies from Regehr's laboratory and others established that PF synapses onto PCS were inhibited by eCBs released during depolarization of the $\mathrm{PC}$ membrane. This depolarization-induced suppression of excitation (DSE) is thus analogous to DSI. Initial experiments by Beierlein and Regehr (2006) examined possible DSE at PF synapses onto SCs and BCs after their depolarization. Neurons voltage clamped at $-70 \mathrm{mV}$ were depolarized to $0 \mathrm{mV}$ for $2 \mathrm{~s}$ while measuring evoked glutamatergic PF EPSCs. As previ- 
ously described, DSE was seen in the PCs, but for the first time was also demonstrated in both types of cerebellar interneurons (Fig. 1). DSE was not observed in the interneurons during CB1R antagonist $N$-(piperidin-1-yl)-5-(4-iodophenyl)-1-(2, 4-dichlorophenyl)-4-methyl-1 H-pyrazole-3-carboxamide (AM251) application [Beierlein and Regehr (2006), their Fig. 1 (http://www.jneurosci.org/cgi/content/ full/26/39/9935/F1)], or in mice lacking the CB1R. Although these data demonstrated retrograde $\mathrm{eCB}$ activation of CB1Rs, the magnitude of DSE was smaller in the interneurons when compared with PCs. To determine whether this resulted from differential sensitivity of CB1Rs on $\mathrm{PF}$ inputs to these neuron subtypes, or from different levels of eCB release, the effects of WIN55,212-2 on PF EPSCs was measured [Beierlein and Regehr (2006), their Fig. 2 (http://www.jneurosci.org/ cgi/content/full/26/39/9935/F2)]. However, EPSCs measured in PCs and interneurons were equally sensitive to the agonist, suggesting that differences in the magnitude of DSE likely resulted from lower levels of eCB released from the interneurons, rather than differences in CB1R sensitivity to eCBs. This suggested that PCs may more tightly regulate their inputs from PFs by releasing higher levels of eCB, compared with SCs and BCs.

An additional strength of this study was the use of physiologically relevant stimulation to release eCBs. Although it is well documented that large, sustained depolarization can release eCBs, it is unlikely that similar neuronal activity occurs under nonpathological conditions. The authors used brief (10 pulses) highfrequency $(50 \mathrm{~Hz})$ trains of electrical stimulation that elicited a synaptically evoked brief suppression of excitatory synaptic transmission (SSE). Similar to DSE, SSE was blocked by AM251 and by the calcium chelator BAPTA, added to the recording pipette. SSE was also blocked by diacylglycerol lipase (DAGL) inhibitors, either included in the pipette [THL (tetrahydrolipstatin)], or in the extracellular solution bathing the cerebellar slice (RHC-80267 [1,6-bis(cyclohexyloximinocarbonylamino)hexane]) [Beierlein and Regehr (2006), their Fig. 3 (http://www. jneurosci.org/cgi/content/full/26/39/ 9935/F3)]. The involvement of DAGL in SSE implicated 2-arachidonylglycerol (2$\mathrm{AG}$ ) as the eCB released from the SCs, because it was known that DAG is converted to 2-AG after its generation via calciumdependent phospholipase $\mathrm{C}$ activation [e.g., after metabotropic glutamate type 1

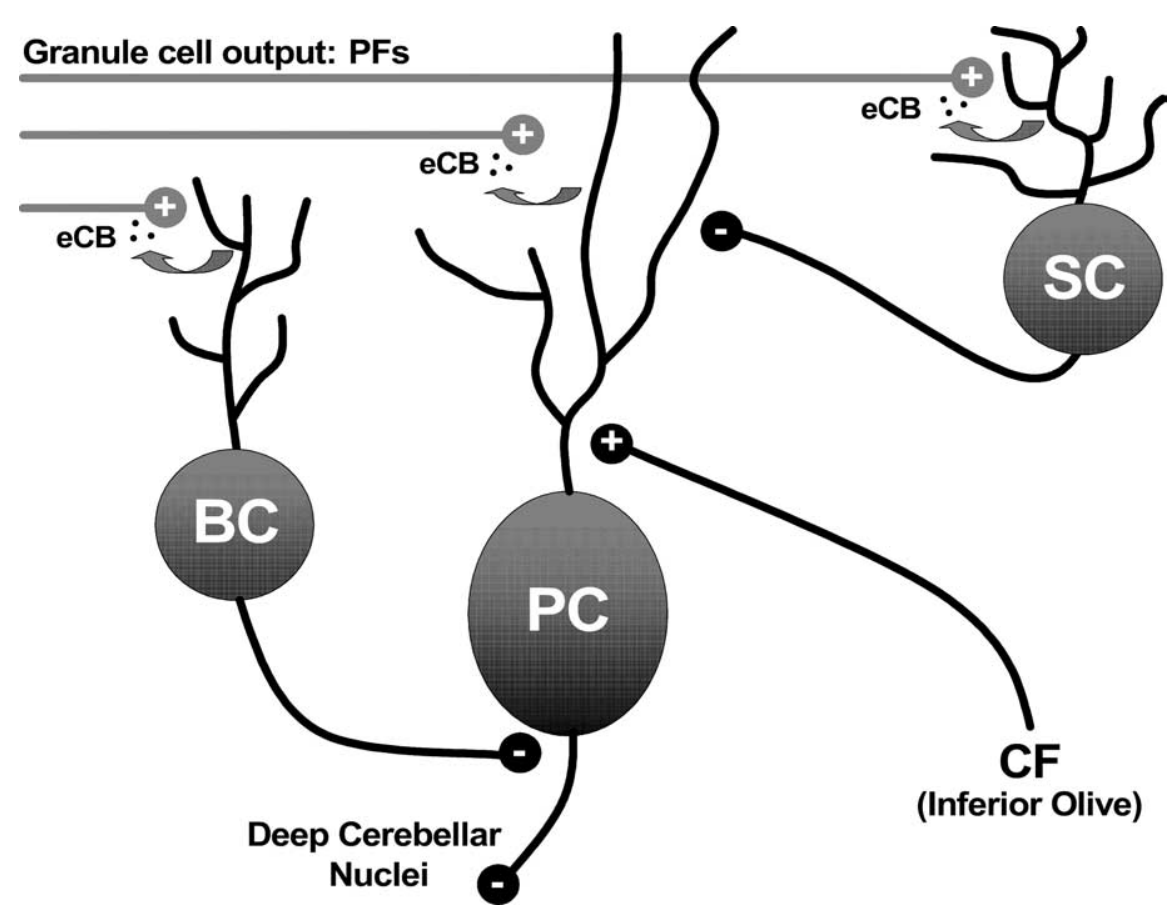

Figure 1. Schematic illustration of postsynaptic eCB release from cerebellar neurons. It was previously shown that PCs could release eCBs in response to glutamatergic PF input. However, the study by Beierlein and Regehr (2006) is the first to show that cerebellar GABAergic BCs and SCs are also able to autoregulate PF inputs through retrograde eCB signaling. This action is expected to reduce the FFI of PCs, thereby increasing the inhibitory PC output to deeper cerebellar nuclei.

(mGluR1) activation] (Stella et al., 1997; Maejima et al., 2005). These data demonstrated that 2-AG was released in the cerebellum under physiologically relevant conditions, and that an increase in postsynaptic calcium and DAGL activation were required.

In addition to the implication of 2-AG in SSE, the authors demonstrated that either mGluR1 or NMDA receptor activation could support SSE in the cerebellum. This was done by examining SSE during the application of the mGluR 1 antagonist CPCCOEt (7-hydroxyiminocyclopropan [b]chromen-1a-carboxylate ethyl ester), or the NMDA receptor (NMDAR) antagonist CPP (2-carboxypiperazin-4-ylpropyl-1-phosphonic acid) [Beierlein and Regehr (2006), their Fig. 4 (http://www. jneurosci.org/cgi/content/full/26/39/ 9935/F4)]. Neither of these antagonists altered SSE alone, but when applied together completely blocked SSE. This implied that the activation of either NMDARs or mGluR1s permitted sufficient accumulation of intracellular $\mathrm{Ca}^{2+}$ to activate DAGL, and that each receptor could independently support 2-AG release from cerebellar SCs. Although mGluR1s and AMPA receptors (Melis et al., 2004; Riegel and Lupica, 2004; Maejima et al., 2005) have been previously implicated in $\mathrm{eCB}$ release, this is the first demonstration that NMDA receptors were involved.
The release of eCBs from SCs and BCs adds an additional level of control of feedforward inhibition (FFI) in the cerebellum. These inhibitory interneurons are thought to function in the cerebellar circuitry to sharpen PF inputs to PCs by temporally limiting this excitatory input. This thereby increases the efficacy of synchronous EPSCs while limiting the effects of succeeding asynchronous inputs by shortening the time frame for synaptic integration to occur (Mittmann et al., 2005). Therefore, it is predicted that, when eCBs are released from these cerebellar interneurons, FFI will be diminished, the duration of PF EPSCs will be prolonged, and the temporal fidelity of this synapse will be diminished. In turn, the expected increase in inhibitory output from PCs to target nuclei (Fig. 1) would alter cerebellar influence on motor learning and behavior.

Together with the results of previous studies (Hoffman et al., 2003; Bacci et al., 2004), the findings of Beierlein and Regehr (2006) emphasize the heterogeneity of eCB release from distinct neuronal phenotypes, and suggests that future studies should seek to determine possible roles for $\mathrm{eCB}$ release from interneurons in other brain regions, as well as the molecular mechanisms that confer this heterogeneity. In turn, this should facilitate our understanding of the mechanisms through which neurons can auto- 
regulate their own activity via the retrograde modulation of synaptic inputs by eCBs.

\section{References}

Bacci A, Huguenard JR, Prince DA (2004) Longlasting self-inhibition of neocortical interneurons mediated by endocannabinoids. Nature 431:312-316

Beierlein M, Regehr WG (2006) Local interneurons regulate synaptic strength by retrograde release of endocannabinoids. J Neurosci 26:9935-9943.

Eccles JC, Ito M, Szentagothai J (1967) The cerebellum as a neuronal machine. New York: Springer.

Hoffman AF, Riegel AC, Lupica CR (2003) Functional localization of cannabinoid recep- tors and endogenous cannabinoid production in distinct neuron populations of the hippocampus. Eur J Neurosci 18:524-534.

Maejima T, Oka S, Hashimotodani Y, OhnoShosaku T, Aiba A, Wu D, Waku K, Sugiura T, Kano M (2005) Synaptically driven endocannabinoid release requires $\mathrm{Ca}^{2+}$-assisted metabotropic glutamate receptor subtype 1 to phospholipase C $\beta 4$ signaling cascade in the cerebellum. J Neurosci 25:6826-6835.

Melis M, Perra S, Muntoni AL, Pillolla G, Lutz B, Marsicano G, Di Marzo V, Gessa GL, Pistis M (2004) Prefrontal cortex stimulation induces 2-arachidonoyl-glycerol-mediated suppression of excitation in dopamine neurons. J Neurosci 24:10707-10715.
Mittmann W, Koch U, Hausser M (2005) Feedforward inhibition shapes the spike output of cerebellar Purkinje cells. J Physiol (Lond) 563:369-378

Riegel AC, Lupica CR (2004) Independent presynaptic and postsynaptic mechanisms regulate endocannabinoid signaling at multiple synapses in the ventral tegmental area. J Neurosci 24:11070-11080.

Stella N, Schweitzer P, Piomelli D (1997) A second endogenous cannabinoid that modulates long-term potentiation. Nature 388:773-777.

Wilson RI, Nicoll RA (2001) Endogenous cannabinoids mediate retrograde signaling at hippocampal synapses. Nature 410:588-592. 